\title{
Wolfgang Däubler
}

\section{Privatautonomie oder demokratische Tarifautonomie?}

\section{Was ist Gegenstand der „Tarifautonomie“?}

Wir sind Tarifverträge über Löhne, Arbeitszeit und sonstige Arbeitsbedingungen gewohnt. Dies bringt die Gefahr mit sich, das Phänomen der Kollektivverträge allein vor dem Hintergrund dieser Erfahrungen zu erklären. Historisch wie rechtsvergleichend ist dies zu eng. Die Gewerkschaft könnte als Verkäufer der Gesamt-Arbeitskraft aller Belegschaftsmitglieder auftreten und als Gegenleistung eine Gesamtsumme aushandeln, über deren Verteilung dann in „Selbstverwaltung" durch die Betroffenen befunden würde. Entsprechende Vorstellungen wurden in den 1920er Jahren in Mexiko entwickelt, ${ }^{1}$ allerdings nicht in die Gesetzgebung übernommen. ${ }^{2}$ Ein ähnlicher Gedanke liegt dem traditionellen „Kameradschaftsgedinge" im deutschen Bergbau zugrunde, wo für eine Gruppenleistung ein bestimmter "Gesamtlohn“ zur Verfügung gestellt wurde. ${ }^{3}$

Eine etwas weniger weitgehende „Vergemeinschaftung“ ergibt sich im kollektiven Arbeitsrecht der USA. Ist eine Gewerkschaft in dem dafür vorgesehenen Verfahren zur Repräsentantin der Belegschaft gewählt worden, so hat sie ein ausschließliches Verhandlungsrecht. Der Arbeitgeber würde sich Sanktionen wegen antigewerkschaftlichen Verhaltens aussetzen, wollte er sich über diese „exclusivity“ hinwegsetzen und mit einzelnen Beschäftigten über Löhne und Arbeitsbedingungen verhandeln. ${ }^{4}$ Inwieweit nach abgeschlossenem Kollektivvertrag mit Rücksicht auf das Günstigkeitsprinzip höhere Vergütungen vereinbart werden können, scheint nicht recht geklärt zu sein; praktisch verhindern lässt sich Derartiges vermutlich nicht. Ein tariflich abdingbares Günstigkeitsprinzip kannte man auch in der Weimarer Zeit ${ }^{5}$ und kennt man noch heute in Österreich, ${ }^{6}$ doch wurde und wird von dieser Ermächtigung zur Nivellierung so gut wie kein Gebrauch gemacht. Im Modell ist freilich die Kollektivität der Vergütungen und der Arbeitsbedingungen sehr viel stärker ausgeprägt als im heutigen deutschen Recht; wer im Kollektivvertrag unter die Räder kommt, kann nicht einmal gemeinsam mit dem Arbeitgeber eine rechtlich abgesicherte Korrektur vornehmen. Dass und mit welcher Intensität sich Gruppen und Individuen in die

1 Lombardo Toledano, La libertad sindical en México, 1926. Dazu Schulenburg, Das kollektive Arbeitsrecht in Mexiko, 1973, S. $50 \mathrm{ff}$.

2 Das mexikanische Arbeitsrecht hat zahlreiche bemerkenswerte Regelungen entwickelt (s. etwa den berühmten Art. 123 der noch heute gültigen Verfassung von 1917, im Wortlaut abgedruckt bei Néstor de Buen, Derecho del Trabajo, 13. Aufl. 2000, p. 348 ff., wo in den Absätzen 12 und 13 die größeren Unternehmen verpflichtet werden, für Wohnraum ihrer Beschäftigten sowie für die Infrastruktur einschließlich Schulen, Krankenhäuser und Erholungsparks zu sorgen), doch bleibt die Realität so fundamental hinter den schönen Ansprüchen zurück, dass auch diese mehr und mehr in Vergessenheit geraten.

3 Dazu etwa Reinhard Hoffmann, Rechtsfortschritt durch gewerkschaftliche Gegenmacht, 1968. Ob die Betroffenen sich immer im Geiste der Solidarität über die Verteilung geeinigt haben, und dies insbesondere in der individualistischer gewordenen Gegenwart tun würden, ist eine offene Frage.

4 Gould, A primer on American Labor Law, 1993, p. 60 et seq.; Getman/Pogrebin, Labor Relations. The Basic Processes, Law and Practice, 1988, p. 96 et seq.

$5 \$ 1$ Abs. 1 Satz 2 TVVO.

$6 \$ 3$ Abs. 1 Satz 2 ArbVG; dazu Firlei DRdA 1981, $1 \mathrm{ff}$ 
Willensbildung der Gewerkschaft einbringen, gewinnt unter diesen Umständen außerordentlich hohe Bedeutung.

Seit dem Erlass des TVG im Jahre 1949 sind uns solche Vorstellungen fremd. Der Tarifvertrag hat als Bezugs- wie als Gestaltungsgröße den einzelnen Arbeitsvertrag, er ist in seinen entscheidenden Teilen „arbeitsvertragsakzessorisch“. Dabei geht es immer nur um die Festlegung eines Mindeststandards, der durch Arbeitsvertrag zugunsten des Arbeitnehmers verbessert werden kann; das Günstigkeitsprinzip gilt nach $\ 4$ Abs. 3 TVG unangefochten. Der Bezug auf den Arbeitsvertrag kennt allerdings einige Ausnahmen; betriebliche und betriebsverfassungsrechtliche Normen sowie Tarifbestimmungen über gemeinsame Einrichtungen reichen über die individualvertragliche Welt hinaus. Sie sind - ebenso wie die skizzierten grundsätzlichen Alternativen - im Hinterkopf zu behalten, wenn es darum geht, wie Tarifverträge konzeptionell zu erfassen sind.

\section{Die These von der kollektiven Privatautonomie}

\section{Stellungnabmen in Rechtsprechung und Literatur}

Eine verbreitete, vielleicht sogar „herrschende“ Auffassung in der juristischen Literatur betrachtet das Zustandekommen und die Wirkung von Tarifverträgen als privatautonomen Vorgang. ${ }^{7}$ Auch die Rechtsprechung des BAG hat diesen Gedanken aufgegriffen. So betonte das Gericht in seiner Entscheidung vom 21.7.20048 $\mathrm{zu}$ den Altersgrenzen von Piloten und in seiner Entscheidung vom 7.6.20069 zur Residenzpflicht, Tarifverträge seien zugleich „Ergebnis kollektiv ausgeübter Privatautonomie“. ${ }^{10}$ Während das BAG auf eine nähere Begründung weitgehend verzichtete, wird in der Literatur darauf hingewiesen, der Tarifvertrag wolle die strukturelle Unterlegenheit des einzelnen Arbeitnehmers überwinden und sichere auf diese Weise die individuelle Regelungsfreiheit der Arbeitsvertragsparteien ab. ${ }^{11}$ Tarifverträge seien "gebündelter Ausdruck individueller Selbstbestimmung “. ${ }^{12}$ Sie würden durch den autonom vollzogenen Verbandsbeitritt legitimiert, durch den sich der Einzelne der Normsetzungsbefugnis der sozialen Gegenspieler unterwerfe. ${ }^{13}$ Damit soll mehr Freiheit vor dem Staat und dem staatlichen Recht gegenüber begründet werden. Insbesondere ist die Bindung an Grundrechte „gelockert“, da bei Freiheitsrechten lediglich die aus den Grundrechten abzuleitende staatliche Schutzpflicht ins Spiel kommt. ${ }^{14}$ Dies ist auch von einzelnen BAG-Senaten ausdrücklich anerkannt worden. ${ }^{15}$ Gleichzeitig ergeben sich überall da Probleme, wo Tarifnormen Auswirkungen auf die Arbeitsverhältnisse Nicht- oder Andersorganisierter haben. ${ }^{16}$

Grundlegend Bayreuther, Tarifautonomie als kollektiv ausgeübte Privatautonomie, 2005, S. 57 ff.; Dieterich, FS Schaub, 1998, S. 117 ff. Ebenso im Ergebnis Löwisch/Rieble, TVG. Kommentar, 3. Aufl. 2012, Grundl. Rn. 26 ff.; Kempen, in: Kempen/Zachert u. a. (Hrsg.), TVG, 5. Aufl. 2014, Grundlagen Rn. 269; ErfK-Dieterich/Linsenmaier, 14. Auf. 2014, Art. 9 GG, Rn. 55 ff.; grundsätzlich auch Bepler, Gutachten zum 70. DJT.

8 BAG - 7 AZR 589/03, EzA \620 BGB 2002 Altersgrenze Nr. 5 = ZTR 2005, 255.

9 BAG - 4 AZR 316/05, NZA 2007, 343.

10 Tz. 20 der ersten und Tz. 30 der zweiten Entscheidung.

11 Bayreuther (Fn. 7), S. $58 \mathrm{f}$.

12 Dieterich (Fn. 7), S. 121 unter Berufung auf Zachert.

13 Dieterich (Fn. 7), S. 121.

14 Richtig erkannt bei Löwisch/Rieble (Fn. 7), Grundl. Rn. 28.

15 BAG 7.6.2006 - 4 AZR 316/05, NZA 2007, 343 Tz. 30: „Ferner können den Tarifvertragsparteien wegen der fehlenden unmittelbaren Grundrechtsbindung Regelungen erlaubt sein, die dem Gesetzgeber verwehrt sind (Schliemann, FS Hanau S. 577, 587; Dieterich, FS Schaub S. 117, 121)“. 
Rechtsdogmatische Qualifizierungen haben den Sinn, die Rechtsanwendung zu erleichtern, weil sie die hinter einem Regelungskomplex stehenden Grundvorstellungen deutlich machen, was bei der Auslegung wie beim Füllen von Lücken normalerweise von großem Nutzen ist. Elementare Voraussetzung ist dabei, dass eine solche Begrifflichkeit in sich stimmig ist, d.h. zumindest alle unbestrittenen Elemente des fraglichen Sachbereichs widerspruchsfrei erklären kann. Dies ist hier keineswegs der Fall.

Zum einen mag man zwar den Beitritt und das Verbleiben in einer Organisation grundsätzlich als privatautonome Entscheidung qualifizieren, doch ist es in hohem Maße fiktiv, dies als Einverständnis mit allen künftigen Tarifabschlüssen zu werten. Will der Einzelne wirklich auch solche Tarifverträge akzeptieren, gegen die er sich in einer (ggf. notwendig werdenden) Urabstimmung ausdrücklich ausspricht, ist er von vornherein mit all den Einschnitten bei der Vergütung und bei den Arbeitsbedingungen einverstanden, die üblicherweise mit einem Sanierungstarif verbunden sind? Im Gesellschafts- und Verbandsrecht wäre eine so weitgehende Unterwerfungserklärung wegen Unbestimmtheit nicht wirksam; ${ }^{17}$ im AGB-Recht würde sie an fehlender Transparenz scheitern.

Zweitens kann der Gedanke einer kollektiven Privatautonomie nicht erklären, dass auch nicht organisierte Arbeitnehmer (und damit die Mehrheit der Beschäftigten) von Tarifverträgen erfasst werden. Dies ist bei betrieblichen und betriebsverfassungsrechtlichen Normen der Fall, die nach $\$ 3$ Abs. 2 TVG für alle im Betrieb tätigen Arbeitnehmer gelten - Tarifbindung des Arbeitgebers vorausgesetzt. Ein Rauchverbot muss ersichtlich für alle verbindlich sein, und eine tarifliche Regelung, wonach die Kündigung von der Zustimmung des Betriebsrats abhängig ist, darf angesichts der „gewerkschaftsneutralen“ Grundausrichtung der Betriebsverfassung nicht auf Organisierte beschränkt sein. Wird ein Tarifvertrag nach $\ 5$ TVG für allgemeinverbindlich erklärt oder nach $\$ \mathbb{S} 3,7 \mathrm{AEntG}$ durch Rechtsverordnung auf die Außenseiter erstreckt, so gilt der „privatautonome“ Tarifvertrag für die Nichtmitglieder des Arbeitgeberverbands und der Gewerkschaft kraft staatlichen Hoheitsakts. Wird von dieser Möglichkeit kein Gebrauch gemacht und handelt es sich auch nicht um betriebliche oder betriebsverfassungsrechtliche Normen, so wird üblicherweise im Arbeitsvertrag auf den Tarifvertrag verwiesen. Dabei handelt es sich nur dem Anschein nach um eine privatautonome Abmachung, da der Arbeitgeber den Inhalt des Arbeitsvertrags in aller Regel einseitig vorgibt: Die Gleichstellung mit den Organisierten ist in praktisch allen Betrieben, deren Inhaber tarifgebunden ist, eine Selbstverständlichkeit, nicht nur, weil der Arbeitgeber in aller Regel gar nicht weiß, wer Mitglied der Gewerkschaft ist, sondern weil er alle Beschäftigten gleich behandeln will, um so keinen Anreiz für einen Gewerkschaftsbeitritt zu schaffen. Schließlich ist auf $₫ 3$ Abs. 3 TVG zu verweisen, wonach ein Tarifvertrag auch dann fortgilt, wenn der Arbeitnehmer oder der Arbeitgeber aus seinem Verband austritt. Gibt es einen stärkeren Entzug von Legitimation als den Austritt, ist dies nicht die intensivste Form der Rücknahme eines erteilten Mandats? ${ }^{18}$ Ein sofortiger Wegfall der Tarifwirkung wäre bei einer privatautonomen Konzeption konsequent, aber das Gesetz sagt in aller Deutlichkeit das Gegenteil. Ähnliches gilt für die Weitergeltung nach $\$ 613$ a Abs. 1 Satz 2 BGB, für die die privatautonome Legitimation auf Arbeitgeberseite fehlt. 
Drittens stellt der Arbeitskampf einen Fremdkörper in einer „privatautonomen“

Ordnung dar. ${ }^{19}$ Dies wird besonders deutlich, wenn man daran denkt, dass auch ein nicht zum Verband gehörendes Unternehmen mit dem Ziel bestreikt werden kann, einen Anschlusstarif abzuschließen. Der Arbeitnehmeraußenseiter hat zudem das Recht, sich am gewerkschaftlichen Streik zu beteiligen. ${ }^{20}$ Auch wenn er davon keinen Gebrauch macht, wird er automatisch Opfer einer Aussperrung, weil sich diese nicht auf Organisierte beschränken darf. ${ }^{21}$ Schließlich verliert er nach der Lehre vom Arbeitskampfrisiko seinen Vergütungsanspruch, wenn im Betrieb infolge streikbedingt ausbleibender Vorprodukte nicht mehr weiter gearbeitet werden kann. ${ }^{22}$

Viertens hat ein Tarifvertrag in der Betriebsverfassung Folgewirkungen, die den Nicht- oder Andersorganisierten in gleicher Weise wie das Gewerkschaftsmitglied treffen. Ist der Abschluss eines Tarifvertrags „üblich“, können nach $\$ 77$ Abs. 3 Satz 1 BetrVG keine (freiwilligen) Betriebsvereinbarungen mehr geschlossen werden. Nur weil andere z.B. in der Vergangenheit Tarifverträge vereinbart haben, muss auch ein daran in keiner Weise Beteiligter auf den Schutz einer unmittelbar und zwingend geltenden Betriebsvereinbarung verzichten. Noch deutlicher wird dieses Phänomen dann, wenn ein Tarifvertrag im Betrieb effektiv eingreift und ein bestimmtes Sachgebiet abschließend regelt; dadurch entfallen nach dem Eingangssatz des $₫ 87$ Abs. 1 BetrVG die auf dasselbe Gebiet bezogenen Mitbestimmungsrechte des Betriebsrats. ${ }^{23}$ Auch hier geht dem Außenseiter ein Stück Schutz verloren. Dasselbe ist im Übrigen dann der Fall, wenn im Arbeitsvertrag eines Nichtorganisierten auf einen Tarif verwiesen wird: Die in Bezug genommenen Tarifnormen werden nach $₫ 310$ Abs. 4 Satz 1 BGB keiner Angemessenheitskontrolle unterzogen, während wortidentische Vertragsbedingungen sehr wohl der Überprüfung zugänglich wären. Auch hier büßt der Einzelne einen Teil seines allgemeinen Schutzes ein, obwohl er am Zustandekommen des Tarifvertrags in keiner Weise beteiligt war. ${ }^{24}$

Fünftens gibt es eine ganze Reihe von Fällen, in denen von gesetzlichen Schutznormen zu Ungunsten der Arbeitnehmer ausschließlich durch Tarif, nicht aber auf andere Weise abgewichen werden kann. Hier liegt die Annahme einer vom Staat eröffneten Aufgabe sehr viel näher als der Gedanke, kraft autonomer Entscheidung hätten sich die sozialen Gegenspieler für eine Verschlechterung des gesetzlichen Status quo entschieden. ${ }^{25}$

\section{Auswirkungen auf die Grundrechtsbindung}

Die weitestgehenden Konsequenzen hat die privatautonome "Theorie“ für die Bindung der Tarifparteien an Grundrechte. Das BAG nahm über mehrere Jahrzehnte hinweg eine volle Bindung der Tarifparteien an die Grundrechte ihrer Mitglieder an, ${ }^{26}$ so dass zum Teil schon von Gewohnheitsrecht die Rede war. ${ }^{27}$ In neuerer Zeit wird zwischen Gleichheits- und Freiheitsrechten differenziert. Bei den ersteren soll es im Ergebnis bei der unmittelbaren Bindung bleiben, da 
es sich bei Art. 3 Abs. 1 GG um ein „fundamentales Rechtsprinzip“ jeder Normsetzung handle. ${ }^{28}$ Dies provoziert die Frage, weshalb Freiheitsrechte wie die Gewissens- oder die Meinungsfreiheit nicht denselben „fundamentalen“ Charakter haben sollen. ${ }^{29}$ Dazu kommt, dass sich viele Fragen sowohl freiheits- als auch gleichheitsrechtlich lösen lassen. Man denke nur an die Fälle des islamischen Kopftuchs, die sich als potentielle Beschränkung der Religionsfreiheit, ${ }^{30}$ aber auch als Diskriminierung wegen der religiösen Überzeugung qualifizieren lassen. ${ }^{31}$ Soll es wirklich einen Unterschied machen, ob der Anwalt im Verfahren den Schwerpunkt auf Art. 4 Abs. 1 GG oder auf $\$ \mathbb{S} 1,7$ Abs. 2 AGG legt? Im ersten Fall hätte er zumindest unklug, im zweiten jedoch recht umsichtig gehandelt. Doch wie verhält es sich, wenn ein kluger Richter nach dem Grundsatz ,iura novit curia" beide Herangehensweisen in seine Überlegungen einbezieht? Volle oder abgeschwächte Bindung?

Werden Freiheitsrechte durch Tarifnormen eingeschränkt, lässt sich das erst dann beanstanden, wenn der Eingriff so weit geht, dass die staatliche Schutzpflicht ausgelöst wird. ${ }^{32}$

Aus der Sicht des einzelnen Koalitionsmitglieds ist der Abschluss eines Tarifvertrags jedoch nicht mit dem Abschluss eines Individualvertrags zu vergleichen, wo die Schutzpflichtlehre zu Recht ihren Platz hat. Vielmehr sieht sich das Individuum der tariflichen Normsetzung in vergleichbarer Weise „ausgeliefert“ wie der Rechtssetzung durch den Staat oder eine Kommune: Er hat sich damit abzufinden. Die demokratische Binnenstruktur des Normgebers reicht als Mittel des Freiheitsschutzes hier wie dort nicht aus - ganz abgesehen davon, dass sich die potentiellen Einflussmöglichkeiten bei der tariflichen Normsetzung immer nur auf eine Seite des Verhandlungstisches beschränken. Das Argument, man könne sich durch Austritt der Tarifwirkung entziehen, ist für die Arbeitnehmerseite völlig fiktiv, da die Arbeitsverträge in aller Regel pauschal auf die einschlägigen Tarifverträge verweisen. Soll man vom einzelnen Arbeitnehmer verlangen, seinem Arbeitgeber eine Änderungskündigung auszusprechen mit der Maßgabe, dass keine Verweisung auf den Tarif mehr erfolgen dürfe, aber gleichzeitig eine Verschlechterung des Gesamtniveaus ausgeschlossen sei? Das geht meilenweit an der Realität vorbei. Auch bei einem Arbeitsplatzwechsel innerhalb derselben Branche wird der Arbeitnehmer bei einem neuen Arbeitgeber wenig Gegenliebe finden, wenn er statt des Tarifs seine eigenen Vorstellungen realisieren möchte. Für die Arbeitgeberseite hat die Austrittsalternative mehr Realitätsgehalt, doch sorgen die Nachbindung gemäß $₫ 3$ Abs. 3 TVG und die Nachwirkung nach $\ 4$ Abs. 5 TVG dafür, dass ein Ausscheren nur mittelfristig wirksam wird. Wenn der führende Vertreter der These von der „kollektiven Privatautonomie“ und der durch sie vermittelten reduzierten Grundrechtsbindung meint, es treffe zu, dass die Adressaten tariflicher Regelungen ihre Rechtsstellung als „fremdbestimmt“ betrachten und betrachten können, doch handle es sich aus der Sicht der Verfas-

So insbesondere BAG 27.5.2004 - 6 AZR 129/03, NZA 2004, 1399. Im Ergebnis übereinstimmend BAG 8.12.2011 - 6 AZR 319/09, AP Nr. 5 zu $\$ 6$ TVÜ: Tarifnormen dürfen nicht zu gleichheits- oder sachwidrigen Differenzierungen führen und deshalb Art. 3 GG verletzen. Ebenso Krause, in: Jacobs/Krause/ Oetker/Schubert, Tarifvertragsrecht, 2. Aufl. 2013, $\mathbb{1}$ Rn. 41.

29 Ähnlich Hensche, in: Däubler/Hjort/Schubert/Wolmerath (Hrsg.), Arbeitsrecht. Handkommentar, 3. Aufl. 2013, Art. 9 GG Rn. 94 (Im Folgenden: HK-ArbR-Bearbeiter).

30 So BAG 10.10.2002 - 2 AZR 472/01, NZA 2003, 483, bestätigt durch BVerfG 30.7.2003 - 1 BvR 792/03, NZA 2003, 959.

31 Näher dazu und zu vergleichbaren Fällen Däubler, GS Zachert, 2010, S. $227 \mathrm{ff}$.

32 BAG 27.5.2004-6 AZR 129/03, NZA 2004, 1399; dazu Dieterich RdA 2005, 177, beide m.w.N. Ähnlich Krause, in: Jacobs/Krause/Oetker/Schubert, Tarifvertragsrecht, $\$ 1$ Rn. 40: grundrechtlicher Mindestschutz. 
sung um grundrechtlich legitimierte Freiheitsausübung, ${ }^{33}$ so muss man sich die

Frage stellen, ob angesichts der zu Recht konstatierten Realität nicht der gewählte Ausgangspunkt eines „privatautonomen Handelns“ der Revision bedarf. Soll wirklich ein Vorgang als „privatautonom“ qualifiziert werden, der nicht nur objektiv fremdbestimmt ist, sondern auch von den handelnden Personen so gesehen wird? Warum soll ein solches Handeln - von allen sonstigen Bedenken einmal abgesehen - geeignet sein, die an sich bestehende Grundrechtsbindung zu lockern? Die gemeinsam handelnden Tarifparteien sind eine „soziale Gewalt“, gegen die der Einzelne des Schutzes bedarf. ${ }^{34}$

Die gelockerte Grundrechtsbindung führt überdies zu erheblichen Wertungswidersprüchen. Einigkeit besteht darüber, dass gegenüber Außenseitern, die nach $\ 3$ Abs. 2 oder nach $\ 5$ TVG vom Tarifvertrag erfasst werden, volle Grundrechtsbindung besteht, da sie sich ja nicht privatautonom der Normsetzungsbefugnis der sozialen Gegenspieler unterworfen haben. ${ }^{35}$ Dies hat zur Folge, dass dann, wenn ein Tarifvertrag für allgemeinverbindlich erklärt wird, sich zwar die Außenseiter, nicht aber die Organisierten darauf berufen könnten, eine einzelne Tarifvorschrift greife übermäßig in ihr Grundrecht z.B. auf Meinungs- oder Gewissensfreiheit ein. Eine solche gespaltene Bindung führt zu einer Benachteiligung wegen Gewerkschaftszugehörigkeit, die durch Art. 9 Abs. 3 Satz 2 verboten ist. Dem in der Weise abzuhelfen, dass für diesen Fall auch eine unmittelbare Bindung an die Grundrechte der Organisierten angenommen wird, ${ }^{36}$ gibt der Allgemeinverbindlichkeit eine Wirkung, für die sie nicht gedacht ist. Auch kann auf diese Weise ein Anreiz für die Arbeitgeberseite entstehen, einer Allgemeinverbindlicherklärung lieber nicht näher zu treten, weil sonst ggf. einzelne Tarifbestimmungen wegen Verstoßes gegen Arbeitnehmergrundrechte rechtswidrig werden könnten. Ähnliche Verwerfungen sind bei betrieblichen und betriebsverfassungsrechtlichen Tarifnormen denkbar. Will man auch dort keine gespaltene Grundrechtsanwendung akzeptieren, weil sonst Art. 9 Abs. 3 GG verletzt wäre, und deshalb gleichfalls zur unmittelbaren Grundrechtsbindung zurückkehren, so hätte man das Problem, dass die Grenzlinie zwischen Inhalts- und Betriebsnormen nicht immer eindeutig zu ziehen ist. Auch wäre man mit der Frage konfrontiert, wie man verfahren soll, wenn eine Tarifbestimmung gleichzeitig Inhalts- und Betriebsnorm ist. ${ }^{37}$ Die Zusatzprobleme, die die privatautonome Erklärung der Tarifnormen schafft, würden schließlich durch die Frage ergänzt, wie eine verfassungskonforme Auslegung von Tarifnormen beschaffen sein soll: eine strenge bei der Einbeziehung von Außenseitern und eine „laxere“, wenn sich die Tarifwirkungen auf die Organisierten beschränken?

Der Eindruck, dass die These von der gelockerten Grundrechtsbindung zu vielen Unklarheiten, Ungereimtheiten und Widersprüchlichkeiten führt, wird zusätzlich durch Probleme der Rechtsanwendung untermauert. Bei wie viel Freiheitsverlust ist der „Interventionspunkt“ erreicht, der die Schutzpflicht auslöst? Kann beispielsweise jede Nebentätigkeit durch Tarifvertrag verboten werden, oder ist das im Hinblick auf das Grundrecht der Berufsfreiheit nicht mehr „hinnehmbar"? Die meisten Freiheitsrechte sollen allerdings eh der tariflichen Regelungsmacht entzogen sein, da sie dem Privatleben zuzuordnen seien. ${ }^{38}$ Doch wie ver-

35 Löwisch/Rieble (Fn. 7), $₫ 1$ Rn. 588; Schiek, in: Däubler (Hrsg.), Tarifvertragsgesetz. Kommentar, 3. Aufl. 2012, Einl. Rn. 235; Schliemann, FS Hanau, 1999, S. 577, 588 f.

36 Schiek, in: Däubler (Hrsg.), Tarifvertragsgesetz, Einl. Rn. 235.

37 Zu solchen Fällen s. Reim/Nebe, in: Däubler (Hrsg.), Tarifvertragsgesetz, $\mathbb{1}$ Rn. 355.

38 ErfK-Dieterich, 11. Aufl. 2011, Einl zum GG Rn. 53; anders nunmehr ErfK-Schmidt, 14. Aufl. 2014, Einl. zum GG, Rn. 53. 
hält es sich mit der Meinungsfreiheit am Arbeitsplatz? Was geschieht mit Normen, die auch die Freizeit einbeziehen, weil sie einen Zusammenhang mit dem Arbeitsverhältnis unterstellen? An welchem Maßstab ist eine Vorschrift wie $\ 8$ Abs. 1 Satz 2 BAT bzw. \41 TVöD zu messen, die von Angestellten des öffentlichen Dienstes verlangt, sich durch ihr „gesamtes Verhalten“ zur freiheitlichen demokratischen Grundordnung zu bekennen? Oder weiter: Könnte die Berufung auf eine Gewissensentscheidung anders als im Arbeitsvertrag durch Tarif ausgeschlossen werden? Die Liste der Fragen lässt sich verlängern. Die in jahrzehntelanger Rechtsprechung mühevoll erarbeitete relative Orientierungssicherheit wird durch die neue Theorie weitgehend zunichte gemacht. Erst recht könnte sie keine Phänomene einer weitergehenden Kollektivierung erfassen, wie sie in Abschnitt I skizziert wurden.

\section{Zwischenergebnis}

Die Theorie von der kollektiven Privatautonomie stellt keine adäquate Erfassung von Tarifverträgen nach dem TVG dar, weil sie insbesondere das Überwirken von Tarifverträgen auf Nicht- oder Andersorganisierte nicht erklären kann. Sie führt außerdem zum Abbau des Grundrechtsschutzes der Arbeitnehmer und erschwert in vieler Hinsicht die Handhabung des geltenden Rechts. Obwohl man eigentlich erwarten könnte, dass die Privatautonomie-These in einer neoliberalen Umwelt weitestgehende Akzeptanz findet, hat sich in den letzten Jahren eine wachsende Zahl von Autoren gegen sie gewandt. ${ }^{39}$

\section{Rückkehr zur Delegationstheorie?}

Bis in die jüngste Vergangenheit vertrat das BAG einheitlich den Standpunkt, die tarifliche Normsetzung sei der Sache nach Gesetzgebung und deshalb nach Art. 1 Abs. 3 GG an die Grundrechte der Normunterworfenen gebunden. ${ }^{40}$ Auch wurde die Formel verwendet, die Tarifparteien dürften deren Grundrechten keine engeren Grenzen ziehen als der Gesetzgeber, ${ }^{41}$ sie seien also in gleicher Weise wie der staatliche Gesetzgeber an Grundrechte gebunden. Dieser könne nicht mehr Befugnisse übertragen als er selbst habe. Das wurde im Allgemeinen so verstanden, dass die Normsetzungsbefugnis vom Staat an die sozialen Gegenspieler delegiert sei; ${ }^{42}$ man sprach deshalb von der Delegationstheorie.

Auch hier ergaben sich Ungereimtheiten und Widersprüche. Die erste zu umschiffende Klippe war Art. 80 Abs. 1 GG. Er sieht eine Verordnungsermächtigung nur an die Bundesregierung, einen Bundesminister oder eine Landesregie-

ErfK-Franzen, $₫ 1$ TVG Rn. 6 (insbesondere beim Verbandstarif überwiegen die „heteronomen Elemente"); Gamillscheg, (Fn. 18), S. 560 ff.; Greiner, Rechtsfragen der Koalitions-, Tarif- und Arbeitskampfpluralität, 2010, S. 99; HK-ArbR-Hensche Art. 9 GG Rn. 94; Thüsing, in: Wiedemann (Hrsg.), Tarifvertragsgesetz, 7. Aufl. 2007, $\$ 1$ Rn. 56 („Die autonomen Elemente sind in einem mitgliederstarken Arbeitgeberverband und erst recht in einer Massengewerkschaft nicht mehr wirksam. Der Umschlag von der autonomen Unterwerfung zu einer heteronomen Rechtsetzung zeigt sich, wenn Individualund Minderheitsinteressen in Großverbänden nicht ausreichend berücksichtigt werden können."); Wiedemann BB 2013, 1397 ff. Differenzierend Löwisch/Rieble (Fn. 7), Grundl. Rn. 25-29 einerseits (eher für privatautonomen Ansatz) und $\mathbb{1} 1 \mathrm{Rn} .588$ andererseits (unmittelbare Grundrechtsbindung); letztlich unentschieden. Zu neoliberalen Angriffen auf die Tarifautonomie s. insbes. Travlos-Tzanetatos, FS Säcker 2011, S. 277 ff.; Berg/Kocher/Platow/Schoof/Schumann, Tarifvertragsgesetz und Arbeitskampfrecht, 4. Aufl. 2013, Teil 1 Rn. 121.

40 Grundlegend BAG 15.1.1955 - 1 AZR 305/54, AP Nr. 4 zu Art. 3 GG.

41 BAG 21.3.1991 - 2 AZR 616/90, AP Nr. 31 zu $\$ 622$ BGB Tz. 20; BAG 23.1.1992 - 2 AZR 470/91, AP Nr. 37 zu $\$ 622$ BGB.

42 Überblick über den Diskussionsstand bei Gamillscheg (Fn, 18), S. 668. 
rung vor und verlangt außerdem, dass diese nach Inhalt, Zweck und Ausmaß

bestimmt sein müsse. Dem wird die den Tarifparteien erteilte Ermächtigung zur Regelung der Arbeits- und Wirtschaftsbedingungen nicht gerecht, doch blieb der Ausweg, dass man von einer verfassungsunmittelbaren Ermächtigung durch Art. 9 Abs. 3 GG ausgehen konnte, die neben Art. 80 Abs. 1 GG steht. Schwieriger war das Problem zu bewältigen, dass es im Prinzip keine Staatsaufsicht wie z.B. bei der kommunalen Selbstverwaltung gibt. Dass keine Überprüfung der Zweckmäßigkeit von getroffenen Entscheidungen erfolgt, ist unschwer akzeptabel, weil genau dies das Wesen der Autonomie ausmacht. Doch gibt es auch keine Rechtsaufsicht von Amts wegen, sondern nur die Möglichkeit einer Klage durch einen Betroffenen, der sich von einer Regelung benachteiligt fühlt. Würde eine Koalition aus der ihr zugewiesenen Rolle ausbrechen, so würde sie sich anderen Zivilrechtssubjekten gegenüber schadensersatzpflichtig machen; die Einsetzung eines „Staatskommissars“, wie sie bei Kommunen und Hochschulen in solchen Fällen vorgesehen ist, kommt dagegen nicht in Betracht. Entscheidend fällt ins Gewicht, dass die Tarifautonomie grundrechtlich fundiert ist, dass es sich um eine von Art. 9 Abs. 3 GG eröffnete Handlungsmöglichkeit handelt, von der man Gebrauch machen kann, aber nicht muss. ${ }^{43}$ Hiermit lässt sich eine „staatsabgeleitete“ Sicht nicht vereinbaren, weil sie viele Dinge wie z.B. die Möglichkeit einer Einigung der sozialen Gegenspieler oder den Arbeitskampf als unbestrittene Teile des „Systems Art. 9 Abs. 3“ nicht erklären kann. Ähnlich wie die Theorie von der kollektiven Privatautonomie greift auch die Delegationstheorie zu kurz; für ein umfassendes „Auf-den-Begriff-Bringen“ der tariflichen Regelungsbefugnis und der mit ihr verbundenen Folgefragen taugt sie nicht. ${ }^{44}$

\section{Kollektivvertragsantonomie als eigenständiges Rechtsinstitut}

\section{Eine Ausnabmeerscheinung?}

Beide hier vorgestellten Erklärungsansätze unternehmen den im Ergebnis erfolglosen Versuch, die Tarifautonomie samt Folgeproblemen allein „zivilrechtlich“ oder allein „öffentlich-rechtlich“ zu erklären. Beides führt nicht zum Ziel, da es sich um eine Institution handelt, die Elemente aus beiden Rechtsgebieten integriert. Im Arbeitsrecht ist dies keine Seltenheit.

Blickt man ins benachbarte Betriebsverfassungsrecht, so findet man zahlreiche Problemlösungen, die aus dem öffentlichen Recht „entliehen“ sind. Betriebsvereinbarungen gelten beispielsweise unbestrittenermaßen auch für und gegen alle die Arbeitnehmer, die den Betriebsrat nicht mit gewählt haben oder die erst nach der Wahl in den Betrieb gekommen sind, nicht anders als eine kommunale Satzung, die auch die Nichtwähler und die später Zugezogenen erfasst. Noch deutlicher wird dies im Falle der erfolgreichen Anfechtung einer Betriebsratswahl. Die Betriebsvereinbarungen, die der fehlerhaft gewählte Betriebsrat geschlossen hat, sind und bleiben wirksam, ${ }^{45}$ nicht anders als entsprechende Beschlüsse eines Gemeinderats, der anschließend wegen fehlerhafter Wahl neu gewählt werden muss. Auf der anderen Seite werden Betriebsratsbeschlüsse nach allgemeinen zi- 
vilrechtlichen Grundsätzen behandelt; hat ein Nichtberechtigter (etwa ein zu Unrecht eingeladener Nachrücker) mitgewirkt, so sind sie unheilbar nichtig. ${ }^{46}$ Die von der Verfassung verliehene Normsetzungsbefugnis der Tarifparteien ist dem öffentlichen Recht entnommen, während der Abschluss eines Tarifvertrags den bürgerlich-rechtlichen Regeln unterworfen ist, die allerdings einige Modifikationen erleiden. ${ }^{47}$ Art. 9 Abs. 3 GG und das TVG sind deshalb primär aus sich selbst heraus und nicht nach einem privatrechtlichen oder öffentlich-rechtlichen „Modell“ zu interpretieren. Dies schließt es nicht aus, im Einzelfall Regeln des Rechtsgebiets heranzuziehen, mit dem das zu entscheidende Problem die engste Affinität besitzt. So liegt es nahe, bei der Interpretation des normativen Teils eines Tarifvertrags auf die für Gesetze entwickelten Grundsätze zurückzugreifen, ${ }^{48}$ während auf den Beitritt zur Gewerkschaft, der die Tarifbindung zur Folge hat, die bürgerlich-rechtlichen Regeln Anwendung finden. ${ }^{49}$

\section{Die vom Staat anerkannte kollektive Selbstbestimmungsordnung}

Der Tarifvertrag ist ein Mittel für die abhängig Beschäftigten, um auf ihre Arbeits- und Wirtschaftsbedingungen im Prinzip gleichgewichtig Einfluss nehmen, sie mitbestimmen zu können. ${ }^{50}$ Auf diese Weise kann Fremdbestimmung gewissermaßen halbiert und durch ein Stück Demokratie ersetzt werden. ${ }^{51}$ Dies geschieht durch „Kollektivierung“, d.h. durch gemeinsames, organisationsvermitteltes Handeln der Arbeitnehmer. Nur solche Vereinigungen, die den Voraussetzungen des Gewerkschaftsbegriffs entsprechen, die insbesondere von der Gegenseite unabhängig sind, können allerdings diese Funktion erfüllen. Durch Art. 9 Abs. 3 GG, durch das TVG und durch das darauf gestützte Richterrecht anerkennt der Staat diese Aufgabe und stellt seine Möglichkeiten und Mittel zur Verfügung, um Tarifverträge in der Realität durchzusetzen.

Die gleichgewichtige Einflussnahme will die Machtasymmetrie kompensieren, die im einzelnen Arbeitsverhältnis besteht. ${ }^{52}$ Dies ist aber nicht ihre einzige Aufgabe. Vielmehr kann auf viele Arbeitsbedingungen nur kollektiv Einfluss genommen werden, weil arbeitsteilige Zusammenhänge eine individuelle Korrektur ausschließen. Ist der Arbeitsprozess in der Weise organisiert, dass beispielsweise alle zu einem bestimmten Zeitpunkt erreichbar sein oder mit der Arbeit beginnen müssen, so kann dieser Zeitpunkt nur durch kollektive Regelung, nicht durch Individualvertrag (mit-)bestimmt werden. Auch gibt es im Bereich arbeitgeberseitiger Zusatzleistungen häufig vorgegebene Ordnungen, deren individuelle Durchbrechung allenfalls in extremen Ausnahmefällen in Betracht kommen kann. Erst recht wären individuelle Abmachungen bei den oben unter I. skizzierten Formen verstärkter Kollektivierung ausgeschlossen.

47 Einzelheiten bei Reim/Nebe, in: Däubler (Hrsg.), TVG, $\mathbb{1} 1$ Rn. 109, 177 ff. m.w.N.

48 Zusammenfassend BAG 12.9.1984 - 4 AZR 336/82, AP Nr. 135 zu \1 TVG Auslegung; BAG 5.2.2009 -6 AZR 114/08, NZA 2009, 559 Tz. 23; zur Literatur s. die Nachweise bei Brecht-Heitzmann/Zachert, in: Kempen/Zachert (Hrsg.), TVG, Grundl. Rn. $486 \mathrm{ff}$.

49 BAG 22.11.2000 - 4 AZR 688/99, AP Nr. 20 zu $\$ 3$ Verbandszugehörigkeit; aus der Lit. s. Lorenz, in: Däubler (Hrsg.), TVG, $₫ 3$ Rn. 25.

50 Zur Interpretation des Art. 9 Abs. 3 im Lichte der Art. 1 und 20 GG s. Däubler, Das Grundrecht auf Mitbestimmung, 3. Aufl. 1975, S. 174 ff. Ähnlich Berg/Kocher/Platow/Schoof/Schumann (Fn. 39), Teil 1 Rn. 57.

51 Vgl. HK-ArbR-Hensche, Art. 9 GG Rn. 76.

52 So auch BVerfG 26.6.1991 - 1 BvR 779/85, BVerfGE 84, 212, 229: „Tarifautonomie ist darauf angelegt, die strukturelle Unterlegenheit der Arbeitnehmer beim Abschluss von Arbeitsverträgen durch kollektives Handeln auszugleichen und damit ein annähernd gleichgewichtiges Aushandeln der Löhne und Arbeitsbedingungen zu ermöglichen.". 
a) Die Stellung des Außenseiters

Inhalts-, Abschluss- und Beendigungsnormen wirken nur für und gegen Organisierte auf beiden Seiten ( $\mathbb{S} 3$ Abs. 1, 4 Abs. 1 TVG). Der Unterschied zu den zahlreichen Rechtsordnungen, die wie Österreich ${ }^{53}$ oder Frankreich ${ }^{54}$ die Tarifbindung des Arbeitgebers genügen lassen, ist vorwiegend ein rechtskonstruktiver. Die Nichtorganisierten erhalten auch bei uns die tariflichen Leistungen, da in den Arbeitsverträgen üblicherweise auf den Tarif verwiesen wird. Hintergrund ist - wie schon erwähnt - die Tatsache, dass der Arbeitgeber bei der Einstellung in der Regel gar nicht weiß, wer Mitglied der Gewerkschaft ist und auch nicht danach fragen darf. Dazu kommt die grundsätzlichere Erwägung, dass die untertarifliche Behandlung von Nichtorganisierten einen Anreiz zum Gewerkschaftsbeitritt schaffen würde, woran dem Arbeitgeber normalerweise nicht gelegen ist. Lediglich bei Aushilfen oder geringfügig Beschäftigten ist eine Ausnahme denkbar, weil sie auch bei Schlechterstellung häufig keinen Gewerkschaftsbeitritt samt anschließender Geltendmachung tariflicher Rechte in Erwägung ziehen werden. Die Beschränkung der Tarifwirkung auf die Organisierten hätte allerdings dann prinzipielle Bedeutung, wenn nach geltendem Recht die arbeitsvertragliche Erstreckung auf Außenseiter eingeschränkt werden könnte, indem man etwa standardisierte Bezugnahmeklauseln als Verstoß gegen Art. 9 Abs. 3 Satz 2 GG qualifizieren würde. ${ }^{55}$ Der Weg über die qualifizierte Differenzierungsklausel ist durch die Rechtsprechung bis auf weiteres verbaut. ${ }^{56} \mathrm{An}-$ erkannt ist lediglich die einfache Differenzierungsklausel, die die Gewerkschaftsmitgliedschaft zum Tatbestandsmerkmal bestimmter Ansprüche macht. ${ }^{57}$ Sie bewirkt nur dann eine effektive Besserstellung der Organisierten, wenn der Arbeitgeber - etwa im Sanierungsfall - nicht über die finanziellen Mittel verfügt, um auch die Außenseiter in den Genuss der vorgesehenen tariflichen Leistungen zu bringen. Dies ist allerdings eine singuläre Situation.

Die Beteiligung an dem Kollektiv, das eine Verbesserung der Arbeits- und Wirtschaftsbedingungen erstrebt, ist freiwillig. Es liegt im Ermessen des Gesetzgebers, die Nichtorganisierten automatisch an den Ergebnissen teilhaben oder alles von einer arbeitsvertraglichen Bezugnahme abhängen zu lassen. Bei betrieblichen und betriebsverfassungsrechtlichen Normen liegt die Erstreckung von der Sache her nahe. Die Allgemeinverbindlicherklärung ist von bestimmten Voraussetzungen abhängig, die nach der Neuregelung durch das Gesetz zur Stärkung der Tarifautonomi ${ }^{58}$ leichter als bisher zu erfüllen sind. Auf Arbeitnehmerseite ergeben sich dabei keine Grundrechtsprobleme. Ist der „erstreckte“ Tarifvertrag besser als die sonst eingreifende gesetzliche Regelung, liegt keine Beschränkung der Rechte und Interessen der betroffenen Arbeitnehmer vor. Weichen einzelne tarifliche Bestimmungen vom gesetzlichen Standard zum Nachteil der Arbeitnehmer ab, ist die Erstreckung gleichfalls im Ergebnis unbedenklich: Selbst ohne sie wäre über eine arbeitsvertragliche Bezugnahme auf den Tarif dasselbe Ergebnis jederzeit erreichbar. In Rechtsprechung und Literatur ist jedenfalls bisher kein Fall bekannt geworden, in dem ein Tarifvertrag zwar das gesetzliche Niveau verschlechtert, gleichzeitig jedoch ein Nichtorganisierter erreicht hätte, dass er von 
dieser Regelung ausgenommen worden wäre. ${ }^{59}$ Auf Arbeitgeberseite kann sich im Gegensatz dazu insbesondere durch eine Erstreckung im Wege der Allgemeinverbindlicherklärung eine zusätzliche Kostenbelastung ergeben, doch ist diese als eine Art Flankenschutz zugunsten der Selbstbestimmungsordnung gerechtfertigt. ${ }^{60}$ Anders als bei der Theorie von der kollektiven Privatautonomie erweist sich unter diesen Umständen die Stellung des Außenseiters ebenso wenig als Problem wie in demokratisch strukturierten Selbstverwaltungseinheiten; wer den Gemeinderat nicht mitwählt oder erst später zugezogen ist, muss die beschlossenen Satzungen gleichwohl gegen sich gelten lassen.

\section{b) Bindung an Grundrechte}

Die Tarifparteien müssen die Grundrechte der Mitglieder wie der Außenseiter respektieren. Maßgebend hierfür ist nicht der Normcharakter des Tarifvertrags oder eine etwaige staatliche Delegation. Vielmehr sind die gemeinsam handelnden Tarifparteien für den Einzelnen eine „soziale Gewalt“, deren Entscheidungen er faktisch ausgeliefert ist. ${ }^{61}$ Die Möglichkeit, als Mitglied auf einer Seite mitzubestimmen, steht der Grundrechtsbindung genauso wenig entgegen wie im staatlichen Bereich, wo der Gesetzgeber trotz seiner demokratischen Wahl die Grundrechte der Bürger zu beachten hat. Die Tarifparteien sind jedoch keine hoheitlich tätige Instanz, sodass die Grundrechte nur entsprechende Anwendung finden können. ${ }^{62}$

Grundrechte sind allerdings im Regelfall im öffentlichen Interesse einschränkbar, was im hier interessierenden Bereich ohne größere Bedeutung ist. Wichtig können jedoch die Fälle sein, in denen ein Arbeitgeberinteresse für ihre Einschränkung spricht. Dieses einfach wie ein öffentliches Interesse zu behandeln, geht nicht an; der Arbeitgeber könnte sonst die Grundrechte der Arbeitnehmer nach eigenen Vorstellungen, d.h. dadurch, dass er seine Interessen entsprechend weit definiert, nachhaltig beschränken. Er kann aber seine grundrechtlich geschützten Belange geltend machen, beispielsweise seine Pressefreiheit ins Spiel bringen, wenn ein Tarifvertrag die „Linie“ seiner Zeitung umdefinieren wollte. Insoweit hat eine Abwägung stattzufinden, die im konkreten Fall zugunsten des Arbeitgebers ausgehen würde. Eine Sonderrolle nimmt allerdings die Berufsfreiheit des Art. 12 Abs. 1 GG ein: Art. 9 Abs. 3 regelt einen Teilbereich der beruflichen Betätigung von Arbeitnehmern wie von Arbeitgebern: Insoweit ist er lex specialis zu Art. 12 Abs. 1 GG. Es stellt sich also nicht die hin und wieder beschworene Frage, jede Lohnerhöhung sei dahingehend zu überprüfen, ob sie nicht einen unverhältnismäßigen Eingriff in die Berufsfreiheit des Arbeitgebers darstelle. Anders wäre zu entscheiden, wenn durch tarifliche Regelungen die Aufnahme oder Fortführung einer beruflichen Tätigkeit ausgeschlossen würde.

Über den Tarif hinausgehende arbeitsvertragliche Ansprüche bleiben im Übrigen nach $₫ 4$ Abs. 3 TVG sowieso erhalten; dies gilt auch für vortarifliche Abmachungen. Dazu Deinert, in: Däubler (Hrsg.), TVG, \4 Rn. 620. Zum Günstigkeitsprinzip und seiner Bedeutung im Rahmen der Deregulierung s. TravlosTzanetatos, FS Säcker, S. 280 ff.

60 So im Ergebnis auch BVerfG 24.5.1977 - 2 BvL 11/74, BVerfGE 44, 322; BVerfG 15.7.1980 - 1 BvR 24/74, BVerfGE 55, 7.

61 Ebenso Gamillscheg (Fn. 18), S. 669; HK-ArbR-Hensche, Art. 9 GG Rn. 94 a. E.; Däubler, Tarifvertragsrecht, 3. Aufl. 1993, Rn. 416; Schlachter, Jahrbuch des Arbeitsrechts, Bd. 40 (2003), S. 51, 58; D. Ulber, Tarifdispositives Gesetzesrecht im Spannungsfeld von Tarifautonomie und grundrechtlichen Schutzpflichten, 2010, S. 236. im Prinzip auch Löwisch/Rieble (Fn. 7), \$1 Rn. $581 \mathrm{ff}$ 
Art. 9 Abs. 3 GG verpflichtet die sozialen Gegenspieler nicht, sich gerade des Tarifvertrags zu bedienen. Durch die Gewährung der normativen Wirkung hat der Gesetzgeber lediglich einen Anreiz geschaffen, einen solchen Weg zu wählen, doch sind mittlerweile auch „sonstige Kollektivverträge“ in Rechtsprechung und Literatur als verfassungsrechtlich geschütztes Mittel anerkannt. ${ }^{63}$ Sie entfalten keine normative Wirkung, verfügen jedoch über große inhaltliche Flexibilität. Inwieweit sie erstreikbar sind, ist höchstrichterlich noch nicht entschieden, aber zu bejahen, da sie sonst von vornherein auf Ausnahmesituationen beschränkt wären, in denen die Interessen beider Seiten im Ergebnis übereinstimmen. Auch wäre es wenig stimmig, würde man zwar einen Arbeitskampf um den obligatorischen Teil eines Tarifvertrags zulassen, ${ }^{64}$ nicht aber um einen sonstigen Kollektivvertrag, der sich gleichfalls auf Arbeits- und Wirtschaftsbedingungen bezieht. „Sonstige Kollektivverträge" können auch als Mittel eingesetzt werden, um neue Fragen aufzugreifen, deren tarifliche Gestaltbarkeit zweifelhaft erscheint. Dies gilt etwa für die kollektivvertragliche Regelung der Beziehung zwischen Entleiher und Leiharbeitnehmer, ${ }^{65}$ doch ist auch an einen im Ausland praktisch gewordenen Fall zu denken, dass Werkvertragsarbeitnehmer längere Zeit bei einem „Hauptunternehmen“ eingesetzt waren und deshalb die Forderung erheben, von diesem übernommen zu werden. Es ist gut, wenn das Tarifsystem sich für neue Bedürfnisse öffnet; die kollektive Selbstbestimmung muss auch unter sich ändernden Bedingungen möglich bleiben.

\section{Fazit}

Weder der Rückgriff auf die Privatautonomie noch der Gedanke einer staatlichen Delegation kann die Schaffung kollektivvertraglicher Regeln ausreichend erklären. Das Denken in sich ausschließenden Alternativen bringt wenig Erkenntnisfortschritt bei der Handhabung von Rechtsmaterien, die Elemente des öffentlichen wie des privaten Rechts in sich aufgenommen haben. Aussichtsreicher erscheint es, Art. 9 Abs. 3 GG aus sich selbst heraus und als Teil der demokratischen Verfassung zu interpretieren. Das schließt nicht aus, bei bestimmten Fragen Anleihen im öffentlichen wie im Privatrecht zu machen. Das Grundrecht des Art. 9 Abs. 3 GG, das in erster Linie Arbeitnehmer schützt, darf kein Mittel zur Verkürzung von Grundrechten der abhängig Beschäftigten werden. Auch muss es offen bleiben für neue soziale und technische Entwicklungen. 